\title{
Topology optimized low-contrast all-dielectric optical cloak
}

\author{
Andkjær, Jacob Anders; Sigmund, Ole
}

Published in:

Applied Physics Letters

Link to article, DOI:

$10.1063 / 1.3540687$

Publication date:

2011

Document Version

Publisher's PDF, also known as Version of record

Link back to DTU Orbit

Citation (APA):

Andkjær, J. A., \& Sigmund, O. (2011). Topology optimized low-contrast all-dielectric optical cloak. Applied Physics Letters, 98(2), 021112. https://doi.org/10.1063/1.3540687

\section{General rights}

Copyright and moral rights for the publications made accessible in the public portal are retained by the authors and/or other copyright owners and it is a condition of accessing publications that users recognise and abide by the legal requirements associated with these rights.

- Users may download and print one copy of any publication from the public portal for the purpose of private study or research.

- You may not further distribute the material or use it for any profit-making activity or commercial gain

- You may freely distribute the URL identifying the publication in the public portal

If you believe that this document breaches copyright please contact us providing details, and we will remove access to the work immediately and investigate your claim. 


\title{
Topology optimized low-contrast all-dielectric optical cloak
}

\author{
Jacob Andkjær ${ }^{\mathrm{a})}$ and Ole Sigmund \\ Department of Mechanical Engineering, Technical University of Denmark, 2800 Kgs. Lyngby, Denmark
}

(Received 13 August 2010; accepted 22 December 2010; published online 13 January 2011)

\begin{abstract}
A systematic methodology for designing low-contrast all-dielectric cloaks operating in the optical range is presented. Topology optimization is used to find the layout of standard dielectric material that minimizes the norm of the scattered field in the surroundings of the cloak. Rotational symmetries are exploited to optimize for multiple angles based on the solution for a single angle of incidence. For a few discrete angles of incidences (1-4) the cloaking is shown to be nearly perfect in a limited frequency range, and even for a rotational symmetric design, cloak and object appear smaller than the noncloaked object. (C) 2011 American Institute of Physics. [doi:10.1063/1.3540687]
\end{abstract}

With the development of transformation optics, ${ }^{1,2}$ the old dream of a device which when wrapped around an object will render the object invisible to the human eye seems within reach. In order to hide a given object for a specific frequency range in the electromagnetic spectrum, it is necessary to obtain the electromagnetic properties of this so-called cloak and be able to realize these properties. The electromagnetic properties, permittivity and permeability, for a cloak can be derived by the aforementioned transformation optics. $^{1,2}$ However, no materials readily available in nature possess the required extreme properties, and hence this calls for a method to artificially engineer materials that do so. So far microwave and near-optical frequency realizations have been achieved based on the use of metamaterials. ${ }^{3,4}$ The tailored microstructure of such metamaterials has to be much smaller than the wavelength, and this makes it very challenging to realize the desired magnetic properties for optical frequencies. Even though several studies have made approximations to ideal cloaking ${ }^{5-8}$ and all-dielectric carpet cloaking has been demonstrated, ${ }^{9}$ nobody has systematically addressed the intriguing question: "How efficiently can we cloak when using conventional simple isotropic dielectric media readily available in nature?"

In this work we apply gradient-based topology optimization ${ }^{10}$ to find the permittivity distribution for a nonmagnetic cloak that hides an ideal metallic cylinder. Furthermore, the evolution of the optimized designs along with the corresponding cloaking properties is investigated for increasing number of incident angles, i.e., increased symmetry. The presented methodology does not only apply for electromagnetic waves, but can also be used for acoustic cloaks.

An infinitely long and ideal metallic cylinder is illuminated by a uniform monochromatic wave propagating in free space. Due to the invariance of the electromagnetic properties along the cylinder axis, the problem can be formulated in a plane perpendicular to the cylinder axis. A first order absorbing boundary condition ${ }^{11}$ is used as an approximation to the Sommerfeld radiation condition in order to truncate the infinite domain. The ideal metallic cylinder can be described as having a perfect electrically conducting (PEC) condition at the interface to free space. Thus, the computational domain is given in two dimensions using three concentric circles, as shown in Fig. 1. The inner, middle, and outer

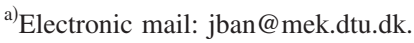

domains represent the PEC cylinder, the cloak, and the surroundings, respectively. The problem is governed by Maxwell's equation in time-harmonic form, and the wave is propagating in simple media. Hence, the presented problem can be reformulated into a scalar Helmholtz equation for the $E_{z}$-polarized wave. We choose to solve the problem using the finite element method. ${ }^{11,12}$ A scattered-field formulation is used in order to reduce the dispersion error. The uniform incident field $E_{z}^{i}$ is formulated as

$$
E_{z}^{i}=E_{0} e^{-j k_{0} \mathbf{k} \cdot \mathbf{r}},
$$

where $E_{0}$ is the amplitude of the wave, $j=\sqrt{-1}, k_{0}$ is the free space wave number, $\mathbf{k}=\left\{k_{x}, k_{y}\right\}^{T}$ is the normalized directional wave vector, and $\mathbf{r}=\{x, y\}^{T}$ is the spatial position vector. In a scattered-field formulation the inhomogeneous Helmholtz equation, the first order absorbing boundary condition ${ }^{11}$ in free space, and the PEC boundary condition are, respectively, formulated as

$$
\begin{aligned}
& \nabla \cdot\left(\mu_{r}^{-1} \nabla E_{z}^{s}\right)+k_{0}^{2} \epsilon_{r} E_{z}^{s}=-k_{0}^{2}\left(\epsilon_{r}-\mu_{r}^{-1}\right) E_{z}^{i}, \\
& \mathbf{n} \cdot \nabla E_{z}^{s}+j k_{0} E_{z}^{s}=0, \\
& E_{z}^{s}=-E_{z}^{i},
\end{aligned}
$$

where $E_{z}^{s}$ is the scattered field; $\epsilon_{r}$ and $\mu_{r}$ are the relative permittivity and permeability, respectively; and $\mathbf{n}=\left\{n_{x}, n_{y}\right\}^{T}$

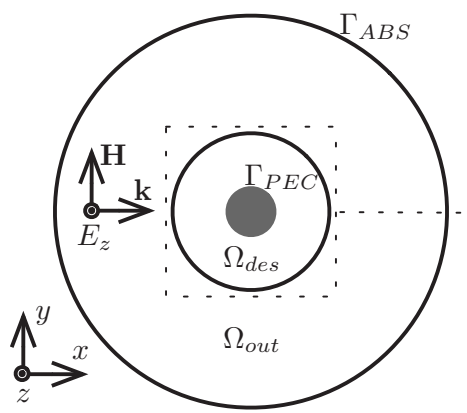

(a)

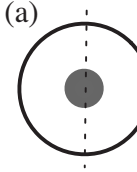

(c)

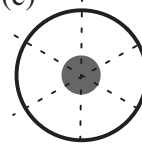

(e)

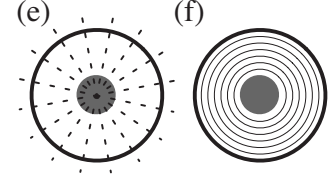

(b)

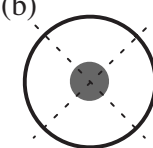

(d)

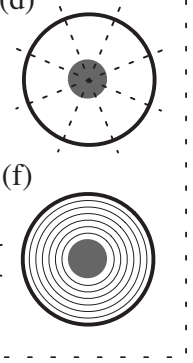

FIG. 1. Computational domain. (a)-(e) show the design domains equipped with one, two, three, four, and eight symmetry lines, respectively. The design domain with rotational symmetry is given in (f). 
is the outward-pointing normal vector of the boundary. The boundary between free space and the cloak is formulated by a continuity condition. In the cloaking literature the boundary between free space and the cloak is usually impedance matched; however, recent studies ${ }^{13}$ have shown that a nonmagnetic cloak whose boundary to free space is not impedance matched also may perform well.

The standard topology optimization approach using a density method is implemented. ${ }^{10}$ The relative permeability is set to 1 in order to mimic a nonmagnetic material; hence, only the spatial distribution of the relative permittivity is varied in the design process. The cloak domain wrapped around the cylinder is defined as the design domain, in which the relative permittivity can be varied continuously on an element basis between 1 and 6 . A design variable, $\rho$ $\in[0 ; 1]$, for each element is introduced as a relative element material density in order to interpolate between free space permittivity $\epsilon_{r}^{\mathrm{min}}=1$ and the upper bound on the permittivity $\epsilon_{r}^{\max }=6$. Thus, the interpolation function is given as

$$
\epsilon_{r}=\epsilon_{r}^{\min }+\rho\left(\epsilon_{r}^{\max }-\epsilon_{r}^{\min }\right) .
$$

In contrast to most topology optimization problems intermediate values of the permittivity in the final design are in this problem both physically sound and realizable as graded index materials, which can be obtained based on varying doping profiles or by nanoperforation. ${ }^{9}$ A density filter ${ }^{14-16}$ is introduced to aid realizations of the optimized designs by enforcing a minimum length scale on the design. Additionally a volume constraint is imposed.

In order to cloak the cylinder the objective is to minimize the norm of the scattered field in the surroundings,

$$
\Phi=\frac{1}{\Phi_{0}} \int_{\Omega_{\text {out }}} E_{z}^{s} \overline{E_{z}^{s}} \mathrm{~d} \mathbf{r},
$$

where the overbar denotes the complex conjugate and $\Phi_{0}$ is the norm of the scattered field in the outer domain when no cloak is present. The reference value $\Phi_{0}$ is included in order to make the objective dimensionless as well as easy to interpret. When $\Phi<1$, cloak and cylinder appear smaller than the noncloaked cylinder. The gradient-based optimization routine, method of moving asymptotes, ${ }^{17}$ is applied to update the design in an iterative approach. The sensitivities are obtained using the adjoint method. ${ }^{18}$

Efficient optimization for multiple incident angles can be performed by introducing symmetry constraints on the design. Symmetry lines divide the original design domain into equally sized subdomains in a number corresponding to the number of incident waves. As the number of angles goes to infinity the design becomes rotationally symmetric as sketched in Fig. 1.

Using the method outlined we have optimized for one, two, three, four, eight, and infinitely many symmetry lines. The results are shown in Fig. 2. When using one and up to four symmetry lines the optimized designs cloak the metallic cylinder nearly perfectly for the considered angles of incidence. The optimized designs for one to four symmetry lines are basically waveguides which guide and delay the waves inside the cloak and phase match them to the waves outside. The angle sweeps shown in Fig. 3 reveal that the cloaking effect is highly localized to the considered angles of incidence for these four designs. In order to make the cloak more
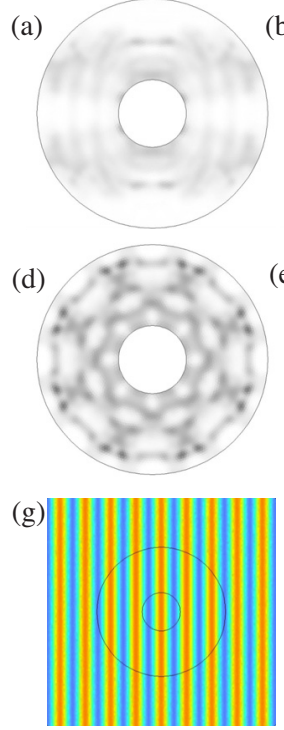

(j)

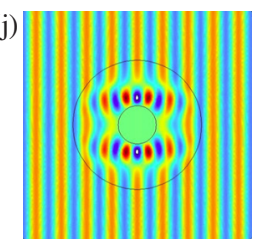

(m)

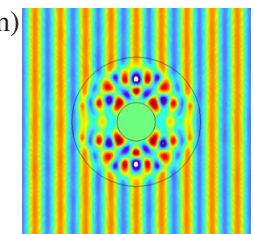

(b)

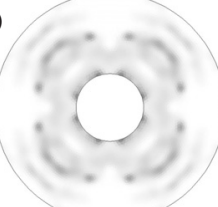

(e)
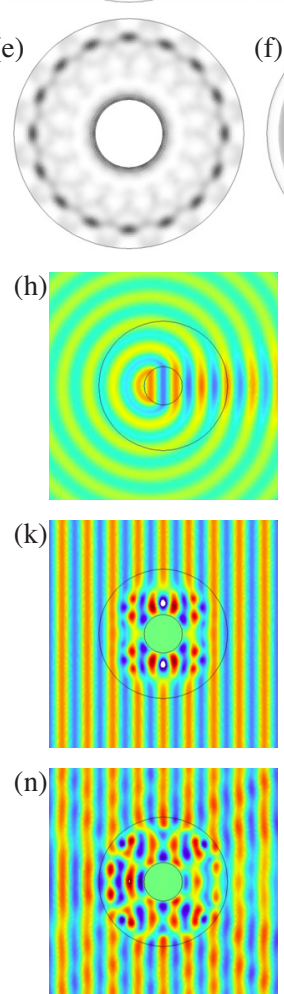

(f)
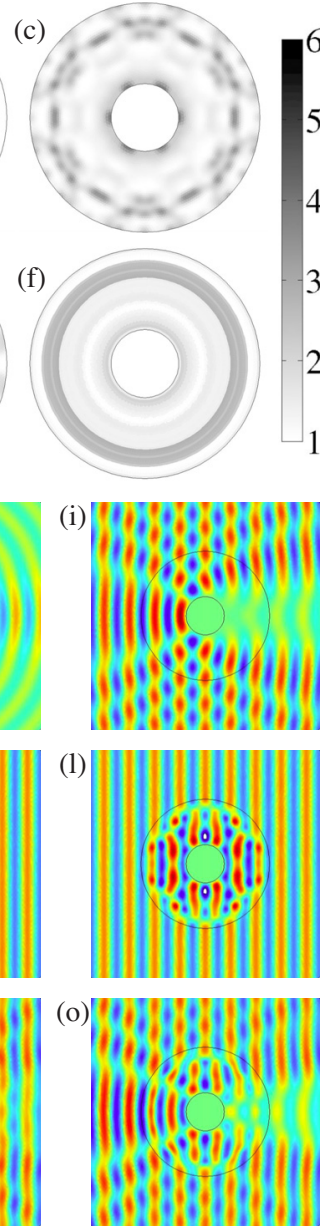

FIG. 2. (Color online) The cloak designs and FE simulations. (a)-(e) The dielectric layout of the cloaks for one, two, three, four, and eight symmetry lines, respectively. (f) The rotational symmetric design. The gray scale indicates the optimized relative permittivity distribution that ranges between 1 and 6. (j)-(o) The corresponding total electric fields. (g)-(i) The incident, scattered, and total electric fields without any cloak wrapped around the PEC cylinder.

versatile to various angles of incidence, more symmetry lines are added to the design domain at the cost of increased objective value as shown in Fig. 4.

A robust design for small perturbations in the angle of incidence is obtained by illuminating with uniform waves incident in an interval from $-5^{\circ}$ to $5^{\circ}$ on the design domain and cylinder. The optimization is formulated as a minimum-

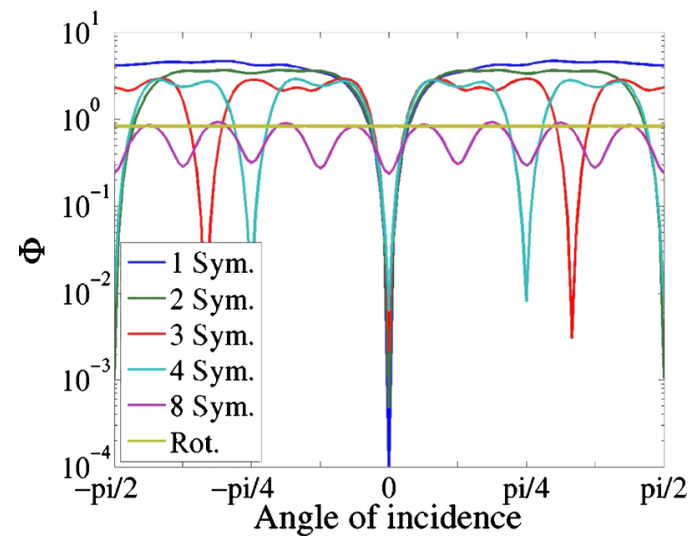

FIG. 3. (Color online) Sweep over the angle of incidence from $-\pi / 2$ to $\pi / 2$. 


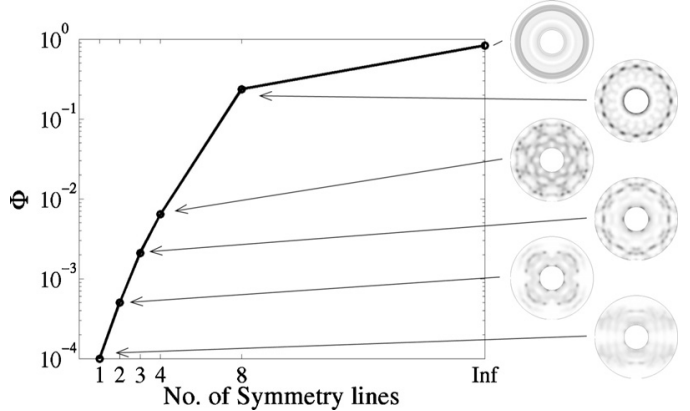

FIG. 4. The evolution of the cloak designs for increasing number of symmetry lines up to a rotational symmetric design. Remark that all designs result in $\Phi<1$, i.e., cloak and cylinder appear smaller than the noncloaked cylinder.

maximum problem, i.e., the load case which yields the highest scattering in each iteration step is minimized. The resulting cloak is shown in Fig. 5(b), and the angle sweep in Fig. 5(a) clearly shows that the cloak can be operated in a broader

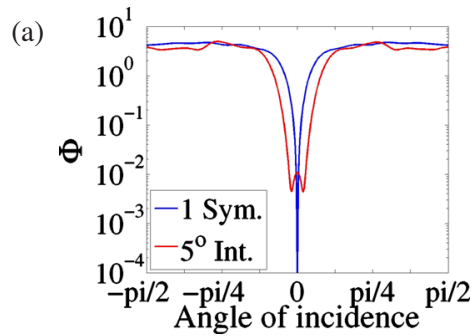

(b)

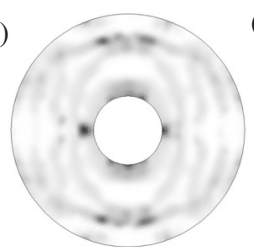

(c)

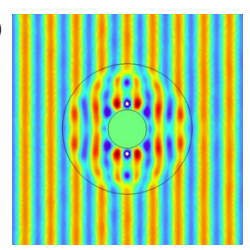

(d)

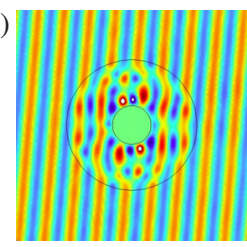

FIG. 5. (Color online) Design, sweep, and finite element simulations of wave incident in an interval from $-5^{\circ}$ to $5^{\circ}$. (a) Sweep through angle of incidence for design optimized at $0^{\circ}$ and in the interval, respectively. (b) The dielectric layout for the cloak operating in the interval. [(c) and (d)] The total electric fields for a wave at $0^{\circ}$ and $-5^{\circ}$ incidence on the cloak from (b), respectively. incident angle interval at the cost of increased scattering.

In conclusion we have outlined a method of designing cloaks that can be realized with readily available dielectric materials. The evolution of topology optimized cloaks operating at various degrees of symmetry is presented. The analysis reveals that near perfect cloaking can be achieved when the cloaks are operating at up to four symmetric angles of incidence. Even for the isotropic design, cloak and cylinder appear smaller than the noncloaked cylinder. However, all presented designs have a narrowband performance, where a deviation in the frequency of less than $10 \%$ will result in $\Phi>1$. The method of designing cloaks can further be extended in several ways, e.g., by optimizing for wider frequency ranges, radar cross sections, or directive properties.

This work was supported by the Eurohorcs/ESF European Young Investigator Award (EURYI) and by the Danish Research Council for Technology and Production (Grant No. 274-06-0507.

${ }^{1}$ J. B. Pendry, D. Schurig, and D. R. Smith, Science 312, 1780 (2006).

${ }^{2}$ U. Leonhardt, Science 312, 1777 (2006).

${ }^{3}$ D. Schurig, J. J. Mock, B. J. Justice, S. A. Cummer, J. B. Pendry, A. F. Starr, and D. R. Smith, Science 314, 977 (2006).

${ }^{4}$ V. M. Shalaev, W. Cai, U. K. Chettiar, H.-K. Yuan, A. K. Sarychev, V. P. Drachev, and A. V. Kildischev, Opt. Lett. 30, 3356 (2005).

${ }^{5}$ B.-I. Popa and S. A. Cummer, Phys. Rev. A 79, 023806 (2009).

${ }^{6}$ W. Cai, U. K. Chettiar, A. V. Kildischev, and V. M. Shalaev, Appl. Phys. Lett. 91, 111105 (2007).

${ }^{7}$ Y. Huang, Y. Feng, and T. Jiang, Opt. Express 15, 11133 (2007).

${ }^{8}$ H. Ma, S. Qu, Z. Xu, and J. Wang, Opt. Express 16, 15449 (2008).

${ }^{9}$ J. Valentine, J. Li, T. Zhentgraf, G. Bartal, and X. Zhang, Nature Mater. 8, 568 (2009).

${ }^{10}$ M. P. Bendsøe and O. Sigmund, Topology Optimization: Theory, Methods and Applications (Springer, Berlin, 2003).

${ }^{11} \mathrm{~J}$. Jin, The Finite Element Method in Electromagnetics (Wiley, New York, 2002).

${ }^{12}$ COMSOL MULTiPHYSICS, COMSOL AB, Stockholm, www.comsol.se.

${ }^{13}$ J. Zhang, Y. Lou, and N. A. Mortensen, Appl. Phys. Lett. 96, 113511 (2010).

${ }^{14}$ B. Bourdin, Int. J. Numer. Methods Eng. 50, 2143 (2001).

${ }^{15}$ T. E. Bruns and D. A. Tortorelli, Comput. Methods Appl. Mech. Eng. 190, 3443 (2001).

${ }^{16}$ O. Sigmund, Struct. Multidiscip. Optim. 33, 401 (2007).

${ }^{17}$ K. Svanberg, Int. J. Numer. Methods Eng. 24, 359 (1987).

${ }^{18}$ J. S. Jensen and O. Sigmund, J. Opt. Soc. Am. B 22, 1191 (2005). 